\title{
Agricultural Mobile Robots in Weed Management and Control
}

\author{
Prakash Kanade \\ Hobby Researcher in Robotics, Artificial Intelligence, IoT, USA \\ Email: Prakashsrs@gmail.com \\ Monis Akhtar \\ Cambridge Institute of Technology, Bangalore \\ Email: monisakhtar0503@gmail.com \\ Fortune David \\ Faculty, Facilitator, LeenaBOT Robotics, Nigeria \\ Email: fortunekbz2009@gmail.com \\ Sunay Kanade \\ Student, LeenaBOT Robotics, Bangalore \\ Email: SunayKanade6@gmail.com
}

\begin{abstract}
The introduction of various robotics technology has made it easier to apply these approaches to agricultural procedures. However, due to the enormous differences in shape, size, rate and type of growth, kind of yield, and environmental needs for different types of crops, implementing this technology on farms has proven difficult. Agricultural processes are a series of time-dependent, methodical, repeated actions. Tilling, soil analysis, seeding, transplanting, crop scouting, insect management, weed removal, and harvesting are all major processes in open arable farming, and robots can help with all of them. By shrinking the range of the search grayscale range, the new method efficiently shortens the algorithm's search speed and reduces computation processing time. The edge contour picture of the corn and weed targets is used as the study object, and we built an algorithm to achieve an accurate selection of the $2 \mathrm{D}$ coordinate points of the corn and weed targets in the field crop image. A quadratic traversal algorithm is proposed in this paper for selecting target $2 \mathrm{D}$ coordinate points in the pixel coordinate system, as well as the related traversal search box. To achieve real-time target recognition and complete automatic cut classification of targets, the Faster R-CNN deep network model based on the VGG-16 feature extraction network is deployed. The use and implementation of our ideas in this study can help intelligent weeding robots perform more precise weeding operations and increase their efficiency.
\end{abstract}

Keywords - Agricultural Robotics, Deep Learning, LeenaBOT, Weeding Robot.

Date of Submission: Sep 02, 2021

Date of Acceptance: Dec 18, 2021

\section{INTRODUCTION}

$\mathrm{W}$ yielding, high-quality crops, and developments in weed control technologies have had a significant impact on agricultural output. Any weed control method that is effective must be both durable and versatile. Despite the variety in field circumstances, robust weed control technologies will successfully manage weeds. Adaptable weed management technology can adapt its method in response to changing weed populations, genetics, and environmental circumstances [1].

Agricultural robotics focuses on crucial work in robotic weeder development, such as weed sensing systems and weed control methods. After a lengthy introduction, the chapter focuses on the issues of robotic weed management, including perception systems that can recognise and categorise weed plants from crop plants, as well as weed control strategies that include chemical and mechanical weed control. [2] Provides a case study of an automated weeding system.
Agricultural robots have a lot of promise in terms of delivering weed control methods that are much more adaptive, even at the plant level. They might be able to target weed plants directly using chemical or horticultural instruments. These features can be seen in agricultural robots because they use current breakthroughs in artificial intelligence (AI) to the control of weeds in crop fields. Bringing $\mathrm{AI}$ and robotics technology to weed management, on the other hand, poses a number of obstacles that, at least in the current state of technology, may restrict the robustness of robotic weed control. Weeds are plants that are out of place or are harmful to the crop plants in the field. Crop plants are cultivated because they have a monetary worth to the grower. As a result, any plant that is not assisting the producer's management scheme, such as volunteer corn growing in a soybean field, might be considered a weed. Plants are classified as weeds based on their location and competition with agricultural plants. As a result, robotic weed control is an ill-posed problem until the agricultural producer's goals for a field are communicated to the robot, which will then recognise and make judgements about which plants are weeds that must be controlled [3-6]. 
Another issue is that, while crop plants are manually planted in a structured manner compatible with agricultural machinery, weed plants emerge and flourish in patterns that are natural to their ecology. As a result, weed plants grow in haphazard patterns on a field. The plants that make up the weed collection in the field are diverse. At different scales, this variability varies as well. The appearance of weed plants varies on a meter-by-meter basis, as well as at larger scales: field, farm, county, state, region, and climatic zone [7].

Weed management solutions take this heterogeneity into account and apply weed control approaches that are both generic and robust enough to control weeds effectively. There are apparent obstacles to overcome in the development of robotic weed management technologies. These difficulties include instructing the robot which plants must be controlled and identifying the unique characteristics of those plants. To keep weed plants under control, their growth must be slowed or stopped without harming surrounding agricultural plants [8].

While there are numerous approaches to weed management, the majority of weed control machines use mechanical or chemical weed control methods. For many years, these methods have been utilised in traditional mechanised agriculture, and they have lately been combined with automation technology to either reduce inputs or impose more accurate control of weed plants. Other weed control strategies exist, such as flame, hot water or steam, or high voltage, but their adoption has been modest, and no study on automating them has been recorded. As a result, only chemical and mechanical weed control strategies will be discussed in this section [9].

When the weeds are growing close to the agricultural plants, it's even more difficult to control them. Figure 1 depicts the five key tasks performed by task-based agricultural robots,

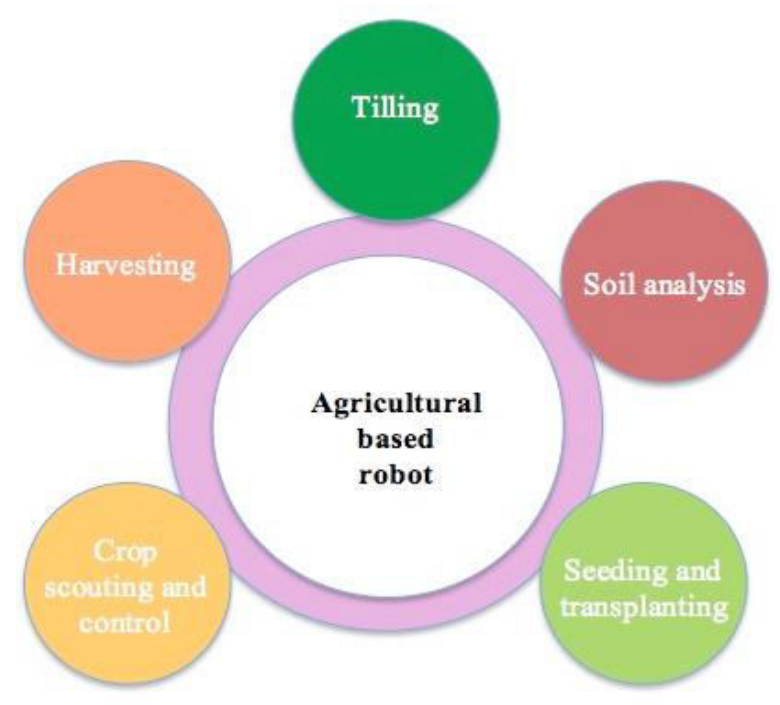

Figure 1. Task-based agricultural robots [10]

\section{LITERATURE SURVEY}

Scholz et al. [16] an automatic soil penetrometer was built and integrated into an autonomous mobile robot named Bonirob. The soil penetrometer features a probing rod with a force sensor that uses a linear actuator to penetrate the soil to a depth of $80 \mathrm{~cm}$. This robot also has surface moisture and temperature sensors, as well as the ability to measure the soil's physical qualities. Their findings demonstrated a significant similarity to data from a commercial penetrologger, with RMSEs of $0.185,0.145$, and $0.120 \mathrm{MPa}$ for loamy sand, sand, and silt soil textures, respectively.

Pobkrut \& Kercharoen et al. [17] To assess specific chemical qualities of soil, researchers created a soilsensing survey robot based on an electronic nose. TGS 825 for hydrogen sulphide; MQ2 for combustible gas; MQ5 for LPG and natural gas; MQ135 for ammonia, benzene, and carbon dioxide; TGS 2600 for air pollutants; and TGS 2602 for volatile organic compound (VOCs) and odorous gases. To receive data from the sensors and manage the entire system, an Arduino Mega 256 controller was used.

Chapman et al. [18] A pheno-copter, or autonomous robotic helicopter for plant phenotyping, was developed. To evaluate images in numerous spectra, this equipment was fitted with two digital cameras and one far infrared camera. One of the tests used a pheno-copter at a height of 60 metres to estimate the ground cover of hybrid sorghum and investigate the link between the number of plants per plot and the green cover for 100 plots. Using data from visible and thermal cameras, the canopy temperature and relative transpiration index in sugarcane were calculated under various irrigation settings. Approximation was used to calculate the prospective transpiration index for 40 sugarcane clones based on green cover and relative crop temperature. Images from an NIR filtered camera were combined with information on longitude, latitude, elevation, and flight log to create a point cloud elevation model, from which the canopy height was calculated.

Polder et al. [19] A totally enclosed, manually propelled platform with a diffused fluorescent lamp and a multispectral camera was designed (RGB \& NIR). As an image of each tulip plant is obtained, the platform is manually moved over the plant. Images in the near-infrared region aid in segmenting the image and distinguishing the plant from the dirt. Fisher's linear discriminant classification techniques are used to identify diseased plants amid healthy plants. The outcome is then compared to the results of an enzyme-linked immunosorbent assay (ELISA) and an expert survey. The findings of this study revealed that agricultural experts correctly recognized 80 percent of ill plants and misclassified healthy plants $10 \%$ of the time. The machine vision system, on the other hand, accurately recognized over $90 \%$ of the infected plants and misclassified $10 \%$ of the healthy plants as diseased. The author also made suggestions about how to improve this platform for robotics.

Griepentrog et al. [20] To construct an autonomous mechanisation system, a Hakotrac 3000 was equipped 
with a GNSS for navigation and an electro-hydraulic valve for steering (AMS). Interfacing with a data logging system that maintained maps for seeding using a grid seeder and punch plater was used to establish crops. The GNSS system was utilised to precisely place seeds in the field. The experimental results revealed a mean standard deviation of $2.53 \mathrm{~mm}$, with 95 percent of the data falling within $5.1 \mathrm{~mm}$ based on a normal distribution.

Hossain and Ferdous et al. [21] The bacterial foraging optimization (BFO) technique was used to create a new algorithm. They also looked at how BFO could be used in mobile robot navigation to find the shortest path between the present position and the goal position in an unknown environment with moving obstacles.

Contrerascruz et al. [22] To overcome the problem of mobile robot path planning, an evolutionary strategy was presented. The suggested method combines the artificial bee colony algorithm as a local search operation with the evolutionary programming algorithm to refine the feasible path discovered by a series of local procedures. The approaches they presented above are mostly for robotic path design, and the majority of them overlooked the fact that weed eradication also necessitates path planning.

Xu et al. [23] For row crops, researchers developed a real-time weed location and variable-speed herbicide spraying (VRHS) system. They suggested an improved particle swarm optimization (IPSO) approach for segmenting wild cornfield weed photos, which improves on the traditional particle swarm optimization algorithm to fulfil field management's real-time data processing needs. Using typical machine learning methods, the aforementioned researchers were able to recognise and segment weeds. They did not, however, provide a method for accurately locating weeds. In order to shorten the length of the trip and the time it takes to complete it.

Liu et al. [24] to identify crop and weed targets, an on-site image spectrometer system was created. Using a small number of spectral bands, multicategory differentiation between weeds or crops and weeds can be achieved. In general, several of the algorithms or specialised systems created above have produced useful experimental findings, but none of them have qualitatively assessed and quantified the distance between crops and weeds, as well as weeds and weeds. Furthermore, the above research was missing the weed eradication path planning guidelines for protecting target crops. This study presents an effective quadratic traversal method for the field weeding robot to tackle the aforesaid challenges as well as provide efficient and accurate weed removal guidance.

\section{METHODOLOGY}

The system structure for agricultural mobile robots for cornfield weeding is depicted in Figure 1. The proposed system's detailed function introduction is as follows. The depth camera is used to extract real-time RGB colour images from the video stream. It's also used to achieve multitarget depth range and path planning for a weeding path that's as efficient as possible. Target recognition and grayscale image processing are two of the most important aspects of data preprocessing. Target recognition and automatic cutting are utilised for corn and weed images, respectively, and grayscale image processing is based on the EXG approach to generate grayscale images in the RGB colour space.

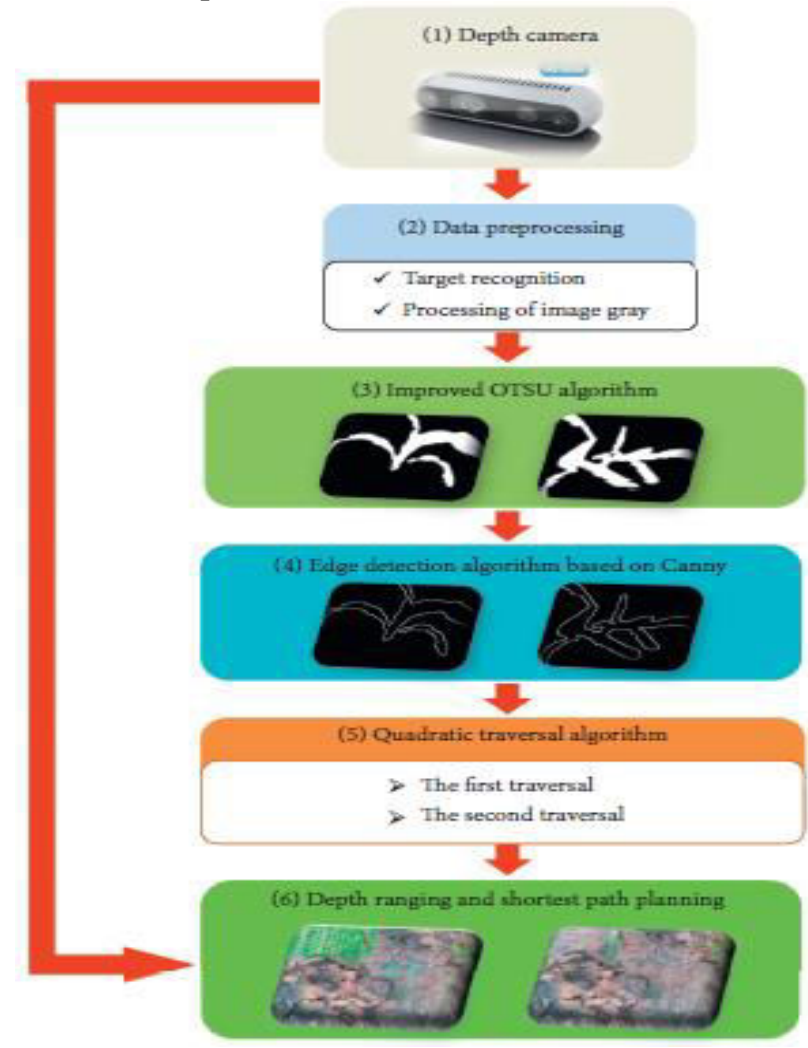

Figure 2. Overview of the system framework

Using our improved OTSU algorithm can achieve the generation and optimization of binary images. Compared with the traditional OTSU algorithm, the algorithm compresses the range of the search grayscale interval. The search speed of the algorithm is effectively improved, and the proposed path planning calculation is time efficient. It meets the demand of the real-time data processing requirements, which allows that our method can be further applied to the mobile agricultural weeding robot in the field.

\section{QUADRATIC TRAVERSAL ALGORITHM}

The edge contour picture of the corn and weed targets is used as the research object in order to achieve an accurate selection of the $2 \mathrm{D}$ coordinate points of the corn and weed targets in the field crop image. A quadratic traversal algorithm is proposed in this paper for selecting target 2D coordinate points in the pixel coordinate system, as well as the related traversal search box. The following are the main phases in implementing the algorithm:

Step I: Define a traversal search box size of pi pixels, a row step size of qj pixels, and a row step size of pi pixels. In the target contour edge picture of size $\mathrm{M} \mathrm{N}$, calculate the number of row direction traversal search boxes and the number of column direction traversal search boxes. 
Step II: The priority row traversal method is used to explore the edge contour image of corn and weed objects using the traversal search box. Store the number of pixels that fulfil the set conditions in the database $\mathrm{C}$ in order for the traversal search box.

Step III: Obtain the serial number for the traversal search box in the database with the maximum number of pixels that matches the stated conditions. It's worth noting that the serial number is a positive integer that starts at one.

Step IV: Using the appropriate serial number of the traversal search box, calculate the position information of the traversal search box on the edge contour image of the target.

\section{EXPERIMENTAL SETUP}

The global positioning system is abbreviated as GPS. It is an omnidirectional, all-weather, all-time, high-precision satellite navigation system capable of providing low-cost, high-precision three-dimensional position, speed, and accurate timing navigation information to users all over the world. The Lidar (VLP-16) is in charge of creating a realtime $2 \mathrm{D}$ or $3 \mathrm{D}$ navigation map of the cornfield at close range, as well as giving real-time $3 \mathrm{D}$ point cloud information around it, which may be used to offer precise navigation information for the cornfield mobile robotics platform. A pair of left-eye and right-eye stereo infrared cameras, infrared dot-matrix laser emitters, and RGB cameras make up the RGB-D depth camera [25].

The size is $90 \mathrm{~mm} 25 \mathrm{~mm} 25 \mathrm{~mm}$ and is suitable for both indoor and outdoor use. The depth camera is based on the triangulation method for binocular stereo distance measurement, in which a pair of stereo infrared cameras collect depth information from the target and a dot-matrix infrared laser emitter projects certain structural features of light on the target in the visual scene. Color image data is collected using an RGB camera, which can align colour image video streams and depth image video streams. The maximum distance that can be covered is ten metres [26]. It's widely used in research fields like drones, robots, and augmented reality/virtual reality.

Universal robots (UR5) have six rotary joints (degrees of freedom) and can perform automated tasks with a maximum load of $5 \mathrm{~kg}$ as a collaborative robotic arm. It has a maximum working radius of $850 \mathrm{~mm}$. The cornfield mobile robotics platform's mobile carrier is a robotic mobile base (Husky A200). It works with four-wheel drive. The maximum payload is 75 kilograms, and the top speed is 1 metre per second. The workstation is essentially an industrial personal computer because it is a highperformance processing unit [27]. On the one hand, it's used to run the algorithm programme that we created. It is, on the other hand, used to communicate with the key devices mentioned above [11-13].

Run the supporting software development programme based on camera on a Windows 10 workstation and compile and generate image acquisition and target ranging software again. The camera's driver is included in the image acquisition and target ranging software, allowing the depth camera to collect image depth and RGB information at a rate of not less than 20 frames per second (fps). The pixels of the collected image are converted to 640480 pixels at the same time. Its goal is to calculate the distance between corn and weeds, as well as between weeds and weeds, using the image depth information currently available, and then plan the shortest weeding path. Figure 3 depicts the specifics.

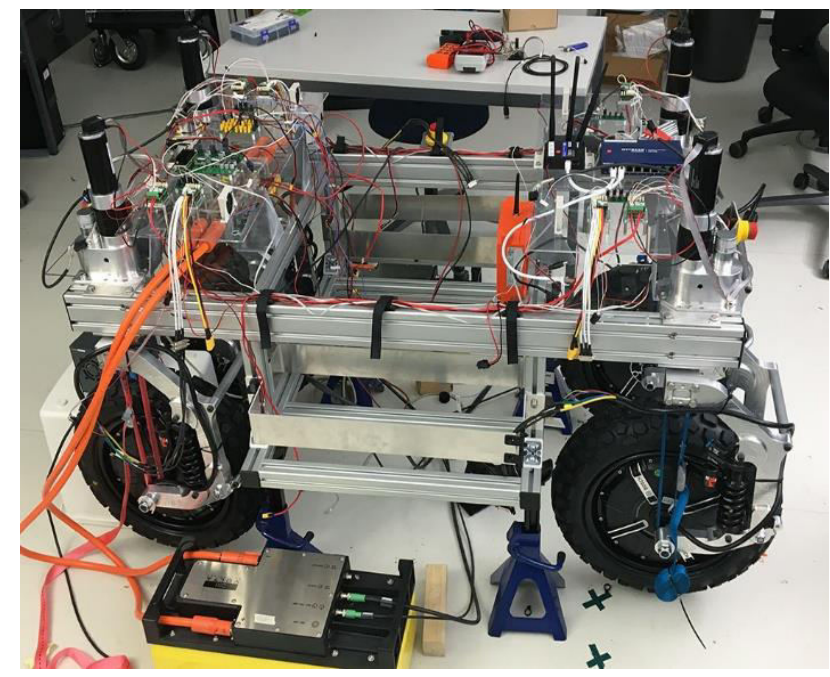

Figure 3. Schematic design of the overall structure with the weeding robot for corn-weed detection.

Data Collection and Preprocessing: The corn field data for this experiment was collected, and our data collection days in 2020 are June 5 to June 9. This is based on the time of seeding and corn growth. Our collection time was dependent on a strong visibility, which was for 03 hours in the morning and afternoon, in order to ensure a clear image collection. Images of corn and weeds in natural settings were taken in three directions: head-up, top-view, and 45degree squint, with the collection steps strictly followed. A high-definition digital camera was used to collect image data [28]. A total of 4102 corns were collected with weed images. All of the images are JPEGs with a resolution of 54723648 pixels. Some of the corn and weed images collected in the agricultural experimental field for various numbers of corn and weeds [14-15].

\section{EXPERIMENTAL RESULTS AND EVALUATION}

Data Preprocessing Results Figure 4 shows the results of corn and weed recognition, as well as automatic weed removal, in data preprocessing. 


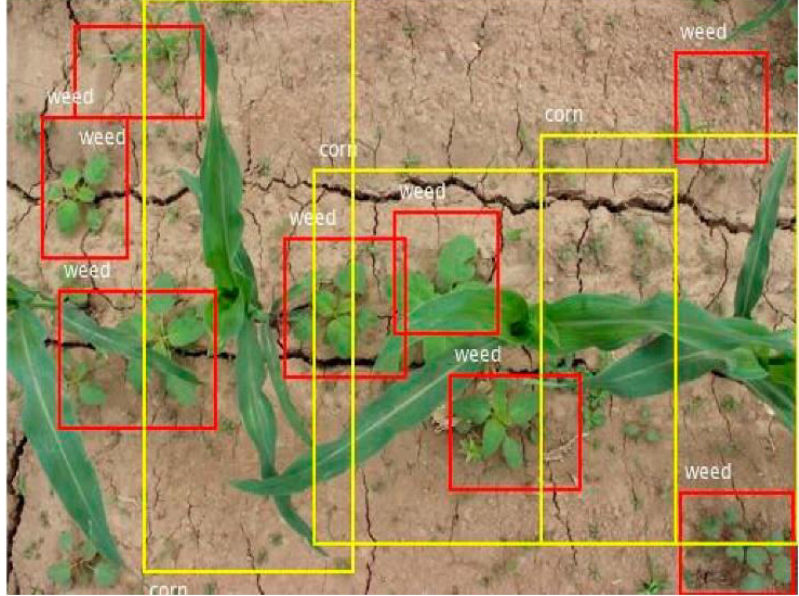

Figure 4. Schematic diagram of corn and weed recognition as well as automatic weed removing.

\section{Novelty of the proposed method and unique feature}

- Using our quadratic traversal algorithm which can achieve the generation and optimization of binary images. The search speed of the algorithm is effectively improved, and the proposed path planning calculation is time efficient.

- Lidar (VLP-16) is responsible for constructing a realtime 2D or 3D navigation map of the cornfield at close range and providing real-time $3 \mathrm{D}$ point cloud information around it, which can further provide precise navigation information for the cornfield mobile robotics platform.

- Therefore, the various performances of our proposed method can basically meet the real-time processing requirements of agricultural weeding robots using our proposed quadratic traversal algorithm.

\section{Comparison between the proposed and the existing one}

\begin{tabular}{|l|c|c|c|}
\hline Algorithms & $\begin{array}{l}\text { Number } \\
\text { of targets }\end{array}$ & $\begin{array}{l}\text { Running } \\
\text { time (s) }\end{array}$ & $\begin{array}{l}\text { Diversity } \\
\text { rate (\%) }\end{array}$ \\
\hline $\begin{array}{l}\text { Path planning } \\
\text { algorithm }\end{array}$ & 7 & 8.63 & 32.4 \\
\hline $\begin{array}{l}\text { Quadratic } \\
\text { traversal } \\
\text { algorithm }\end{array}$ & 7 & 9.10 & 3.90 \\
\hline Genetic algorithm & 7 & 4.20 & 18.3 \\
\hline
\end{tabular}

RCNN is considered, though others are available

Thee Faster R-CNN deep network model based on the VGG-16 feature extraction network is used to realize realtime target recognition and complete automatic cut classification of targets. By returning the predicted parameter information of the border regression and the color of the prediction border, the target category in the image can be accurately determined.

\section{CONCLUSION}

The task of weed one-time removal operations in cornfields is investigated in this study. To achieve realtime target recognition and complete automatic cut classification of targets, the Faster R-CNN deep network model based on the VGG-16 feature extraction network is deployed. The target category in the image can be accurately determined by returning the predicted parameter information of the border regression and the color of the prediction border, and we realized the data connection between deep learning and traditional algorithms. The use and implementation of our ideas in this study can help intelligent weeding robots perform more precise weeding operations and increase their efficiency. In the meantime, it has significant practical implications for promoting the use of intelligent weeding robots in the field.

\section{FUTURE ENHANCEMENT}

The following are two aspects of our future work: (1) A quantitative analysis of the robot's power consumption. (2) Take into account the impact of outdoor dynamic environmental factors.

\section{REFERENCES}

[1] Prakash Kanade, Ashwini P, "Smart Agriculture Robot for Sowing Seed," International Journal of Engineering Science and Computing (IJESC), vol. 11, no. 01, Pages. 27563-27565, 2021.

[2] Prakash Kanade, Jai Prakash Prasad, "Arduino based Machine Learning and IoT Smart Irrigation System", International Journal of Soft Computing and Engineering (IJSCE), vol. 10, no. 4, Pages. 1-5, 2021.

[3] Marinoudi, V.; Sørensen, C.G.; Pearson, S.; Bochtis, D. Robotics and labour in agriculture. A context consideration. Biosyst. Eng. 2019, 184, 111-121. [Google Scholar] [CrossRef]

[4] Pedersen, S.M.; Fountas, S.; Have, H.; Blackmore, B.S. Agricultural robots-System analysis and economic feasibility. Precis. Agric. 2006, 7, 295-308. [Google Scholar] [CrossRef]

[5] Prakash Kanade, Jai Prakash Prasad, "Machine Learning Techniques in Plant Conditions Classification and Observation," IEEE 2021 5th International Conference on Computing Methodologies and Communication (ICCMC), 2021, pp. 729-734, doi: 10.1109/ICCMC51019.2021.9418386.

[6] Pedersen, S.M.; Fountas, S.; Sørensen, C.G.; Van Evert, F.K.; Blackmore, B.S. Robotic seeding: Economic perspectives. In Precision Agriculture: Technology and Economic Perspectives; Pedersen, S.M., Lind, K.M., Eds.; Springer International Publishing: Cham, Switzerland, 2017; pp. 167-179. ISBN 978-3-319-68715-5. [Google Scholar]

[7] Blackmore, B.S.; Fountas, S.; Gemtos, T.A.; Griepentrog, H.W. A specification for an autonomous 
crop production mechanization system. In Proceedings of the International Symposium on Application of Precision Agriculture for Fruits and Vegetables, Orlando, FL, USA, 1 April 2009; Volume 824, pp. 201-216. [Google Scholar]

[8] H. Wang, W. Mao, G. Liu, X. Hu, and S. Li, "Identification and location system of multi-operation apple robot based on vision combination," Transactions of the Chinese Society of Agricultural Machinery, vol. 43, pp. 165-170, 2012.

[9] M. Montalvo, J. M. Guerrero, J. Romeo, L. Emmi, M. Guijarro, and G. Pajares, "Automatic expert system for weeds/crops identification in images from maize fields," Expert Systems with Applications, vol. 40, no. 1, pp. 75-82, 2013.

[10]R. Bogue, "Robots poised to revolutionise agriculture," Industrial Robot: An International Journal, vol. 43, no. 5, pp. 450-456, 2016.

[11] O. Bawden, J. Kulk, R. Russell et al., "Robot for weed species plant-specific management," Journal of Field Robotics, vol. 34, no. 6, pp. 1179-1199, 2017.

[12] M. Guerrero, M. Guijarro, M. Montalvo et al., "Automatic expert system based on images for accuracy crop row detection in maize fields," Expert Systems with Applications, vol. 40, no. 2, pp. 656664, 2013

[13] Haug S, Michaels A, Biber P, Ostermann J, 2014. Plant classification system for crop/weed discrimination without segementation. IEEE Winter Conf on Application of Computer Vision, March. pp: 1142-1149.

[14] Horowitz J, Ebel R, Ueda K, 2010. No-till. Farming is a growing practice. USDA Econ Inform Bull 70 . November.

[15] Jensen K, Nielsen SH, Jorgensen RN, Bogild A, Jacobsen NJ, Jorgensen OJ, Hansen CLJ, 2012. A low cost, modular robotics tool carrier for precision agriculture research. Proc Int Conf on Precision Agriculture; July.

[16] Jeon HY, Tian LF, 2009. Direct application end effector for a precise weed control robot. Biosyst Eng 104: 458-464. https://doi.org/10.1016/j.biosystemseng.2009.09.005

[17] Kargar AHB, Shrizadifar AM, 2013. Automatic weed detection system and smart herbicide sprayer robot for corn fields. RSI/ISM Int Conf on Robotics and Mechatronics; February. pp: 13-15.

[18] Prakash Kanade, Prajna Alva, Jai Prakash Prasad and Sunay Kanade, "Smart Garbage Monitoring System using Internet of Things(IoT)," IEEE 2021 5th International Conference on Computing Methodologies and Communication (ICCMC), 2021, pp. 330-335, doi: 10.1109/ICCMC51019.2021.9418359.

[19] Canny, A. 1986. A computational approach to edge detection. IEEE Trans. Pattern Anal. 8:769698.Google ScholarPubMed

[20] Scholz C, Moeller K, Ruckelshausen A, Hinck S, Goettinger M, 2014. Automatic soil penetrometer measurements and GIS based documentation with the autonomous field robot platform bonirob. Int Conf on Precis Agr; July.

[21] Prakash Kanade, Prajna Alva, Sunay Kanade, Shama Ghatwal, "Automated Robot ARM using Ultrasonic Sensor in Assembly Line," International Research Journal of Engineering and Technology (IRJET), vol. 07, no. 12, Pages. 615-620, 2020.

[22] Pobkrut T, Kerdcharoen T, 2014. Soil sensing survey robots based on electronic nose. Int Conf on Control, Automation and System. pp: 1604-1609. https://doi.org/10.1109/ iccas.2014.6987829

[23] Chapman S, Merz T, Chan A, Jackway P, Hrabar S, Dreccer M, Holland E, Zheng B, Ling T, JimenezBerni J, 2014. Pheno-copter: A low-altitude, autonomous remote-sensing robotic helicopter for high-throughput field-based phenotyping. Agronomy 4: 279-301. https://doi. org/10.3390/agronomy4020279

[24] Polder G, van der Heijden GWAM, van Doorn J, Baltissen TAHMC, 2014. Automatic detection of tulip breaking virus (TBV) in tulip fields using machine vision. Biosyst Eng 117: 35-42. https://doi.org/10.1016/j. biosystemseng.2013.05.010

[25] Griepentrog HW, Dühring Jaeger CL, Paraforos DS, 2013. Robots for field operations with comprehensive multilayer control. Künstl Intell 27: 325-333. https://doi.org/10.1007/ s13218-013-0266-z

[26] M. A. Hossain and I. Ferdous, "Autonomous robot pathplanning in dynamic environment using a new optimizationtechnique inspired by bacterial foraging technique," Robotics and Autonomous Systems, vol. 64, pp. 137-141, 2015.

[27] M. A. Contreras-Cruz, V. Ayala-Ramirez, and U. H. Hernandez-Belmonte, "Mobile robot path planning using artificial bee colony and evolutionary programming," Applied Soft Computing, vol. 30, pp. 319-328, 2015.

[28] Y. Xu, Z. Gao, L. Khot, X. Meng, and Q. Zhang, “A real-Time weed mapping and precision herbicide spraying system for row crops," Sensors, vol. 18, no. 12, p. 4245, 2018.

[29] B. Liu, R. Li, H. Li, G. You, S. Yan, and Q. Tong, "Crop/Weed discrimination using a field imaging spectrometer system," Sensors, vol. 19, no. 23, p. 5154, 2019. 